\title{
ROBUST BEAMFORMING FOR INTERFERENCE REJECTION IN MOBILE COMMUNICATIONS *
}

\author{
Jaume Riba, Jason Goldberg and Gregori Vàzquez \\ Department of Signal Theory and Communications. Universitat Politècnica de Catalunya. \\ E.T.S.E.Telecomunicació,Campus Nord,Edifici D5, c/Gran Capità s/n,08034,Barcelona,Spain. \\ Tel: +34 3 4017052; fax: +3434016447 e-mail:\{jriba, jason,gregori\}@gps.tsc.upc.es
}

\begin{abstract}
The problem of robust beamformer design in the presence of moving sources is considered. A new technique based on a generalization of the constrained minimum variance beamformer is proposed. The method explicitly takes into account changes in the scenario due to the movement of the desired and interfering sources, requiring only estimation of the desired DOA. Computer simulations show that the resulting performance constitutes a compromise between interference and noise rejection, computational complexity, and sensitivity to source movement.
\end{abstract}

\section{INTRODUCTION}

The use of spatial diversity in wireless communications has recently received considerable attention [1]-[4]. Specifically, base station antenna arrays combined with adaptive beamforming techniques offer the possibility of increased channel capacity and wider area coverage. The general goal is that of exploiting the spatial dimension (i.e., the fact that co-channel users are located at different points in space) to combat interference, noise, and multipath fading of the desired signal.

In some systems, the beamformer is designed using data taken over some short time interval. The weights are then held constant over some generally longer interval before being updated in response to changes in the scenario. This may be due to the lack of a continually available beamformer reference and/or limited computational resources. Such is the case, for example, in the uplink (mobile to basestation) of temporal reference beamforming (TRB) systems [2]. The training sequence used in the design of the beamformer is only available over a small fraction of the entire data frame length. The resulting beamformer weights are frozen and used for the remainder of the frame in spite of changes in the scenario. Moreover, in both TRB and spatially referenced beamformer (SRB) systems, the use of frozen weights may be desired so as to avoid the additional

This work has been supported in part by EEC Contract HCM/CHRXCT-930405, PRONTIC/CICYT TIC95-1022-C05-01 and CIRIT/Generalitat de Catalunya GRQ93-3021. complexity of continuous updating. Another example is found in time division duplex (TDD) systems when the uplink beamformer is used during the subsequent downlink (basestation to mobile) [5] in an effort to improve reception at the mobile.

The minimum rate at which the beamforming weights are updated depends on the speed with which the scenario changes. The performance of some beamformers can be seriously degraded when this updating rate is not sufficiently high. Consider, for example, the use of the optimum minimum variance beamformer when the additive sensor noise power is low compared to the desired, signal-of-interest (SOI) power which, in turn, is lower than the power of the interfering signals-not-ofinterest (SNOI's). The optimum beamformer will often place sharp, deep nulls at the SNOI directions of arrival (DOA's) in order to maximize the output signal to noise plus interference ratio (SINR). However, output SINR can be severely degraded if source movement is significant relative to the beamformer update rate.

This paper considers the problem of robust beamformer design in the presence of sources that move over the interval during which its weights are held constant. A new method based on a generalization of the minimum variance beamformer employing a stochastic model for the movement of the sources is proposed. The resulting array response pattern possesses broad nulls in the directions of the SNOI's and is therefore less sensitive to changes in the SNOI DOA's. The approach is similar to that presented in [3] for non-moving scattered sources, which requires a full parametric characterization of the scenario (SOI and SNOIs). However we consider the case of point sources with movement and propose a beamformer which requires only DOA estimation of the SOI. Computer simulations have been included to show that the method strikes a good compromise between SINR and robustness in the presence moving sources.

\section{PROBLEM FORMULATION}

\subsection{Model and Optimum Beamforming}

Consider a single narrow band desired signal-of-interest (SOI) in the far-field of an array of $M$ sensors in the 
presence of $N-1$ co-channel SNOI's and additive, white, circular Gaussian noise. The $M$ dimensional snapshot vector at snapshot time index $k$ is modeled as a sum of desired, interfering and noise components:

$$
\begin{aligned}
& \mathbf{y}(k)=\mathbf{d}(k)+\mathbf{i}(k)+\mathbf{n}(k), \\
& \mathbf{d}(k)=\mathbf{a}\left(\theta_{0}(k)\right) s_{0}(k), \quad \mathbf{i}(k)=\sum_{m=1}^{N-1} \mathbf{a}\left(\theta_{m}(k)\right) s_{m}(k) .
\end{aligned}
$$

$\theta_{0}(k)$ and $\theta_{m}(k), m \in\{1, \cdots, N-1\}$, denote the DOA's at time $k$ of the single SOI and the $N-1$ SNOI's, respectively, while a $(\cdot)$ denotes the $M x 1$ steering vector as a function of DOA. $s_{0}(k)$ and $s_{m}(k), m \in\{1, \cdots, N-1\}$, represent the SOI and the $N-1$ SNOI's at time $k$. Lastly, $\mathbf{n}(k)$ denotes the $M x 1$ additive noise vector at time $k$. Assuming that all the sources are mutually uncorrelated, the correlation matrix of the array snapshot is given by:

$$
\begin{aligned}
\mathbf{R}_{y} & =E\left[\mathbf{y} \mathbf{y}^{H}\right]=\mathbf{R}_{d}+\mathbf{R}_{i+n}, \\
\mathbf{R}_{d} & =\gamma_{0} \mathbf{a}\left(\theta_{0}\right) \mathbf{a}^{H}\left(\theta_{0}\right), \\
\mathbf{R}_{i+n} & =\sum_{m=1}^{N-1} \gamma_{m} \mathbf{a}\left(\theta_{m}\right) \mathbf{a}^{H}\left(\theta_{m}\right)+\sigma^{2} \mathbf{I}
\end{aligned}
$$

where $(\cdot)^{H}$ denotes conjugate transpose, and explicit dependence on the time index $k$ has been and will be suppressed where possible. $\gamma_{0}$ and $\gamma_{m}, m \in\{1, \cdots, N-1\}$, denote the power of the SOI and the $N-1$ SNOI's, respectively. $\sigma^{2}$ denotes the sensor noise power. The output SINR is defined as:

$$
\operatorname{SINR}[\mathbf{w}]=\frac{\mathbf{w}^{H} \mathbf{R}_{d} \mathbf{w}}{\mathbf{w}^{H} \mathbf{R}_{i+n} \mathbf{w}}=\frac{\gamma_{0}\left|\mathbf{w}^{H} \mathbf{a}\left(\theta_{0}\right)\right|^{2}}{\mathbf{w}^{H} \mathbf{R}_{i+n} \mathbf{w}} .
$$

The optimum minimum variance beamforming (which also maximizes the SINR) is defined as the solution of the following constrained minimization problem:

$$
\min \mathbf{w}^{H} \mathbf{R}_{y} \mathbf{w} \quad \text { subject to } \quad \mathbf{w}^{H} \mathbf{R}_{d} \mathbf{w}=\gamma_{0} .
$$

As the matrix $\mathbf{R}_{d}$ is of rank one, the optimum beamformer can be expressed simply as $\mathbf{w}_{o}=$ $\mathbf{R}_{y}^{-1} \mathbf{a}\left(\theta_{0}\right) /\left(\mathbf{a}^{H}\left(\theta_{0}\right) \mathbf{R}_{y}^{-1} \mathbf{a}\left(\theta_{0}\right)\right)$. For high signal-tonoise ratio (SNR), relatively low signal-to-interference ratio (SIR), and $N-1<M$, the output SINR will be very high. The array response pattern magnitude, defined by $|G(\theta)|=\left|\mathbf{w}^{H} \mathbf{a}(\theta)\right|$, is such that the SNOI components will probably be almost perfectly nulled: ${ }^{1}$ $\left|G\left(\theta_{m}\right)\right| \approx 0, m \in\{1, \cdots, N-1\}$. Alternatively, for comparable SNR and SIR levels, the optimum beamformer will maximize output SINR by likely attenuating (rather than nulling) the SNOI's to some level beneath that of the SOI.

\footnotetext{
${ }^{1}$ Note, however, that this is less likely to be true for moderate or low SNR when one or more SNOI's are close in angle (relative to the array resolution) to the SOI. This is because a null so close to the SOI can produce high sidelobe levels elsewhere in the response which amplify the additive noise term.
}

\subsection{Problem Statement}

In general, for a beamformer designed to function in a given scenario, subsequent movement of the sources will decrease performance dramatically. This is specially true in those scenarios where an small pointing error caused by the movement of the SOI can lead to signal attenuation effects, and where an small movement of the SNOIs will seriously degrade output SINR because deep nulls are placed at the initial SNOI locations. Although this problem can often be ameliorated by imposing additional constraints on the beamformer to broaden the mainlobe and nulls [6] this solution would require accurate, frequently updated estimates of the SOI and SNOI DOA's, $\left\{\theta_{m}\right\}_{m=1}^{N-1}$. Thus, the problem addressed in this paper is how to broaden the main lobe and the nulls without knowledge of the SNOI DOA's. The resulting beamformer, while offering sub-optimum SINR performance, is to possess greater robustness in the presence of source motion.

\section{EFFECT OF SOURCE MOTION}

To quantify the loss in performance due to source motion, such movement is modeled as a zero mean Gaussian random walk in angle:

$$
\begin{array}{r}
\theta_{m}(k+1)=\theta_{m}(k)+r_{m}(k), \quad m \in\{0, \cdots, N-1\} \\
E\left[r_{m}(k) r_{m^{\prime}}\left(k^{\prime}\right)\right]=\sigma_{r m}^{2} \delta\left(k-k^{\prime}\right) \delta\left(m-m^{\prime}\right) .
\end{array}
$$

It is easy to show that the resulting angle trajectories are then random processes of mean $\theta_{m}(0)$ and timedependent variance $\sigma_{\theta_{m}}^{2}(k)=k \sigma_{r m}^{2}$. A covariance matrix averaged over angle can now be defined as:

$$
\begin{aligned}
\overline{\mathbf{R}}_{y}= & E_{\theta}\left[\mathbf{R}_{y}\right]=\overline{\mathbf{R}}_{d}+\overline{\mathbf{R}}_{i+n} \\
\overline{\mathbf{R}}_{d}= & \gamma_{0} \int p\left(\theta \mid \theta_{0}(0), k \sigma_{r 0}^{2}\right) \mathbf{a}(\theta) \mathbf{a}^{H}(\theta) d \theta \\
\overline{\mathbf{R}}_{i+n}= & \sum_{m=1}^{N-1} \gamma_{m} \int p\left(\theta \mid \theta_{m}(0), k \sigma_{r m}^{2}\right) \mathbf{a}(\theta) \mathbf{a}^{H}(\theta) d \theta \\
& +\sigma^{2} \mathbf{I}
\end{aligned}
$$

where $p(\cdot \mid \cdot, \cdot)$ is the probability density function of a real Gaussian random variable of specified mean and variance. The above expression is identical to that of a spatial channel model used to describe local scattering in mobile communications applications [3][7]. For the $M$ element linear uniform array with inter-element sensor spacing $d,(7)$ may be written as [7]:

$$
\begin{gathered}
\overline{\mathbf{R}}_{y}=\sum_{m=0}^{N-1} \gamma_{m}\left[\mathbf{a}\left(\theta_{m}\right) \mathbf{a}^{H}\left(\theta_{m}\right)\right] \odot \mathbf{Q}\left(\theta_{m}(0), k \sigma_{r m}^{2}\right) \\
+\sigma^{2} \mathbf{I} \\
\mathbf{a}\left(\theta_{m}\right)=\left[1, e^{j 2 \pi d \sin \theta_{m}}, \cdots, e^{j 2 \pi d(M-1) \sin \left(\theta_{m}\right)}\right]^{T} \\
{\left[\mathbf{Q}\left(\theta_{m}(0), k \sigma_{r m}^{2}\right)\right]_{p q}=e^{-2[\pi d(p-q)]^{2} k \sigma_{r_{m}}^{2} \cos ^{2} \theta_{m}(0)}}
\end{gathered}
$$


where $\odot,(\cdot)^{T}$, and $[\cdot]_{p q}$ respectively denote the Schur Hadamard element-by-element matrix product, the vector transpose operation, and the $p q$ th element of a matrix. The corresponding $\overline{S I N R}$ (SINR averaged over angle) for this scenario at time $k$ at the output of a beamformer designed at time $k^{\prime}$ (i.e., which uses data up to time $k^{\prime}$ ) is defined as:

$$
\overline{S I N R}\left[k, \mathbf{w}\left(k^{\prime}\right)\right] \equiv \frac{\mathbf{w}^{H}\left(k^{\prime}\right) \overline{\mathbf{R}}_{d}(k) \mathbf{w}\left(k^{\prime}\right)}{\mathbf{w}^{H}\left(k^{\prime}\right) \overline{\mathbf{R}}_{i+n}(k) \mathbf{w}\left(k^{\prime}\right)}
$$

where $0 \leq k^{\prime} \leq k$. The $\overline{S I N R}$ is expected to decrease as $k-k^{\prime}$ increases (i.e., as more time elapses between when the beamformer is designed and when it is used). The next section proposes a new robust beamformer, $\mathbf{w}_{r}(\cdot)$ which, if designed at time $k^{\prime}=0$ with the intention of being used over the interval $k \in\{0, \cdots, K\}$, offers the following compromise with respect to the optimum beamformer:

$$
\begin{aligned}
& \overline{\operatorname{SINR}}\left[K, \mathbf{w}_{o}\right]<\overline{\operatorname{SINR}}\left[k, \mathbf{w}_{r}\right] \\
& <\overline{\operatorname{SINR}}\left[0, \mathbf{w}_{o}\right]=\operatorname{SINR}\left[\mathbf{w}_{o}\right]
\end{aligned}
$$

where all beamformers are designed at $k^{\prime}=0$. That is, as the sources move with time, the robust beamformer will perform better than the optimum beamformer but at the cost of suboptimum initial performance.

\section{ROBUST BEAMFORMING}

The robust beamformer presented in this section will be based on the source motion model as reflected in the averaged covariance matrix of (10). The effect of the associated "DOA spreading matrix" as defined in (12) is to smear or spread the point sources over intervals centered on the initial $(k=0)$ DOA's. A beamformer which is designed to be robust in presence of source motion should take this smearing effect into account in order to create broad nulls for the interfering users. To this end we can define the optimum robust minimum variance (i.e. maximum $\overline{S I N R}$ ) beamformer as the solution of the following constrained minimization problem:

$$
\min \mathbf{w}^{H} \overline{\mathbf{R}}_{y} \mathbf{w} \quad \text { subject to } \quad \mathbf{w}^{H} \overline{\mathbf{R}}_{d} \mathbf{w}=\gamma_{0}
$$

It is well known that the maximum of (15) can be solved using generalized eigenanalysis:

$$
\begin{aligned}
\overline{\mathbf{R}}_{d} \mathbf{w}_{r} & =\lambda_{\max } \overline{\mathbf{R}}_{y} \mathbf{w}_{r} \\
\mathbf{e} & =\mathbf{e}_{\max }\left[\overline{\mathbf{R}}_{d}, \overline{\mathbf{R}}_{y}\right] \\
\mathbf{w}_{r} & =\mathbf{e} /\left(\mathbf{e}^{H} \overline{\mathbf{R}}_{d} \mathbf{e} / \gamma_{0}\right)^{1 / 2}
\end{aligned}
$$

where $\mathbf{e}_{\max }$ denotes the generalized eigenvector associated with the maximum eigenvalue $\lambda_{\max }$ of the corresponding matrix pencil. This beamformer should have the effect of broadening the potentially sharp nulls that would have been placed over the SNOI's by the ordinary optimum beamformer. The spreading matrix (12) is a function of source DOA at $k=0$ and random walk variance for each source. To avoid estimation of the DOA's of the SNOI's and the random walk variances of all the sources, consider a pessimistic, worst case spreading matrix:

$$
[\mathbf{Q}]_{p q}=e^{-2[\pi d(p-q)]^{2} K \sigma_{r_{\max }^{2}}^{2}}
$$

where $\sigma_{r_{\max }}^{2}$ is an upper bound on the random walk variance. Then the average covariance matrices can be computed as follows:

$$
\begin{aligned}
& \overline{\mathbf{R}}_{d}(K)=\mathbf{R}_{d}(0) \odot \mathbf{Q} \\
& \overline{\mathbf{R}}_{y}(K)=\mathbf{R}_{y}(0) \odot \mathbf{Q} .
\end{aligned}
$$

Finally an estimate of $\mathbf{R}_{d}(0)$ is obtained using an estimate of the SOI angle $\theta_{0}(0)$ (supplied by a DOA tracking algorithm [8]) in (3), and an estimate of $\mathbf{R}_{y}(0)$ is obtained directly using data taken over some interval during which all source angles does not change significantly: $\hat{\mathbf{R}}_{y}(0)=\frac{1}{L} \sum_{k=-L+1}^{0} \mathbf{y}(k) \mathbf{y}^{H}(k)$

We finally note that the proposed robust beamformer involves a norm constraint (15) with respect to the average desired covariance matrix, placed on the weight vector. While the problem of optimization with linear constraints can be solved by using dynamic adaptive methods such as the generalized side lobe canceller (GSLC), the development of adaptive methods to solve the present problem is more difficult. This issue and the dynamic adaptive computation of $\sigma_{r \max }^{2}$ is left for future work.

\section{RESULTS}

Computer simulations results now illustrate the performance of the new technique. Consider $N=4$ sources (one SOI and three SNOI's) impinging on a uniform linear array of $M=8$ sensors with half-wavelength interelement spacing. The initial DOA's of the SOI and the three SNOI's at time $k=0$ are, respectively: $\theta_{0}(0)=0^{\circ}$, $\theta_{1}(0)=25^{\circ}, \theta_{2}(0)=40^{\circ}$, and $\theta_{3}(0)=-35^{\circ}$. The source powers are: $\gamma_{0}=1, \gamma_{1}=\gamma_{2}=\gamma_{3}=20$. The sensor noise power is $\sigma^{2}=0.05$. The resulting SNR's for the four sources are $13 \mathrm{~dB}$ for the SOI and $26 \mathrm{~dB}$ for each of the three SNOI's. The corresponding SINR is $-17 \mathrm{~dB}$. The performance of the proposed beamformer is considered over a period of $K=500$ output samples wherein the sources undergo random walks in angle with time $K$ standard deviation parameters: $\sqrt{K} \sigma_{r 1}=5.1^{\circ}$, $\sqrt{K} \sigma_{r 2}=5.6^{\circ}, \sqrt{K} \sigma_{r 3}=6.7^{\circ}$, and $\sqrt{K} \sigma_{r 4}=6.3^{\circ}$. The new technique is applied to this scenario assuming $\sqrt{K}$. $\sigma_{r_{\max }}$ of (19) is set to $7^{\circ}$. Fig. 1 shows the broad nulls placed by the robust beamformer around the angles of the interferences compared against the sharp nulls created by the conventional optimum beamformer. Fig. 2 compares the average output SINR as a function of time from which the beamformer is designed for the optimum and the robust beamformer. It is seen that although the optimum beamformer designed at time $k=0$ 
yields very high initial performance, this steadily degrades with time as the SNOI's move further away from the nulls present in the array response pattern. On the other hand, the robust beamformer performance remains roughly constant with time, initially somewhat inferior to that of the optimum beamformer designed at $k=0$, but quickly offering greatly improved performance as a function of time due to the wider nulls present in its array response pattern. Fig. 3 shows the effect of the sensor noise power on output SINR at time $k=K$. As expected the robust beamformer performance gain diminishes as input SNR decreases. Lastly Fig. 4 shows the time $k=500$ performance as the initial $(k=0)$ DOA of the first SNOI approaches that of the SOI. As a broad null is created at the SNOI angle, the SOI is also attenuated if the SNOI and SOI angles are near. Therefore the robust beamformer performance gain worsens as the angular separation decreases.

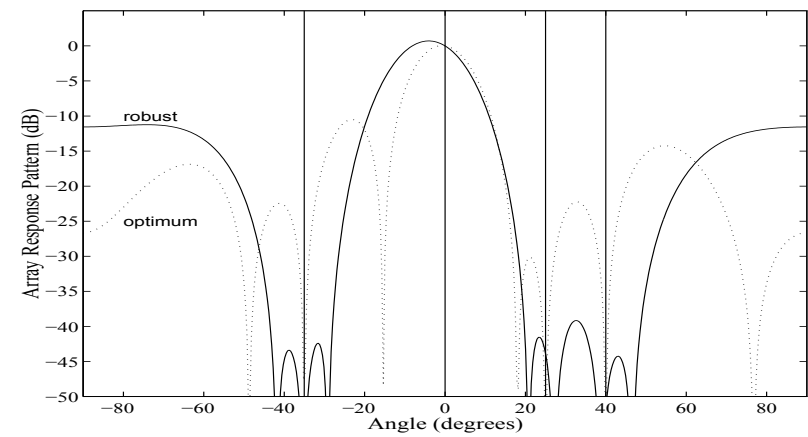

Figure 1: Array response patterns.

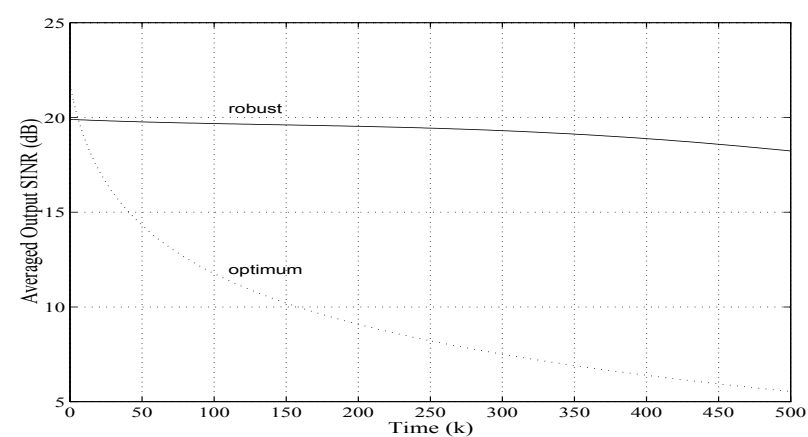

Figure 2: Output SINR vs. time.

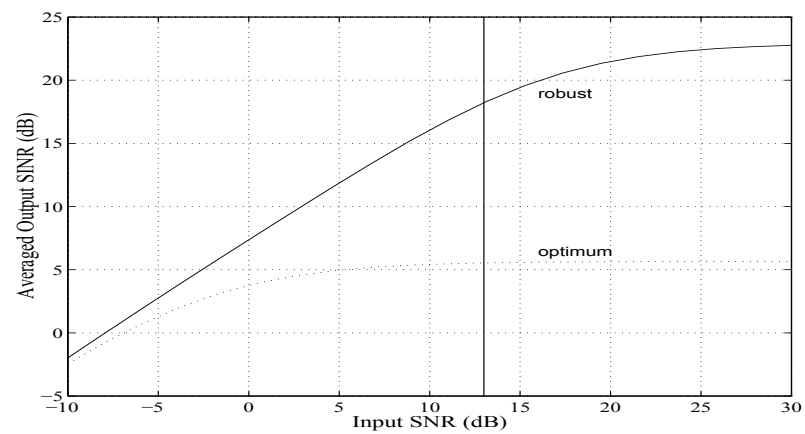

Figure 3: Output SINR vs. SNR.

\section{CONCLUSION}

A new method for the design of beamformers which are robust in the presence of source motion was presented. The technique is useful in applications such as mobile communications where the beamforming weights are frozen for some length of time (despite changes in the scenario) before being updated. Broad null are placed at the angles of arrival of the interfering users without requiring estimation of them. The resulting performance has been shown to constitute a trade-off between output SINR, computational complexity, and sensitivity to source movement.

\section{References}

[1] J.H. Winters. Optimum combining in digital mobile radio with cochannel interference. IEEE Journal on Selected Areas in Communications, vol. 2: pp. 528539, July 1984.

[2] J.H. Winters. Signal acquisition and tracking with adaptive arrays in the digial mobile radio system IS54 with flat fading. IEEE Transactions on Vehicular Technology, vol. 42: pp. 377-384, November 1993.

[3] P. Zetterberg and B. Ottersten. "The spectrum efficiency of a basestation antenna array system for spatially selective transmission". IEEE Transactions on Vehicular Technology, vol. 44: pp. 651-660, August 1995.

[4] B. Ottersten and P. Zetterberg. "Basestation antenna array in mobile communications". to appear in Proc. of "Tyrrehenian International Workshop on Digital Communications", Viareggio, Italy, 1995.

[5] W.C. Jakes. Microwave Mobile Communications. Wiley, 1974.

[6] D.H. Johnson and D.E. Dudgeon. Array Signal Processing-Techniques and Concepts. Prentice Hall, 1993.

[7] T. Trump and B. Ottersten. "Estimation of nominal direction of arrival and angular spread using an array of sensors". submitted to Signal Processing, 1994.

[8] J.Riba, J. Goldberg, and G. Vàzquez. "Signal selective doa tracking for multiple moving targets". to appear in Proc. IEEE ICASSP, May 1996.

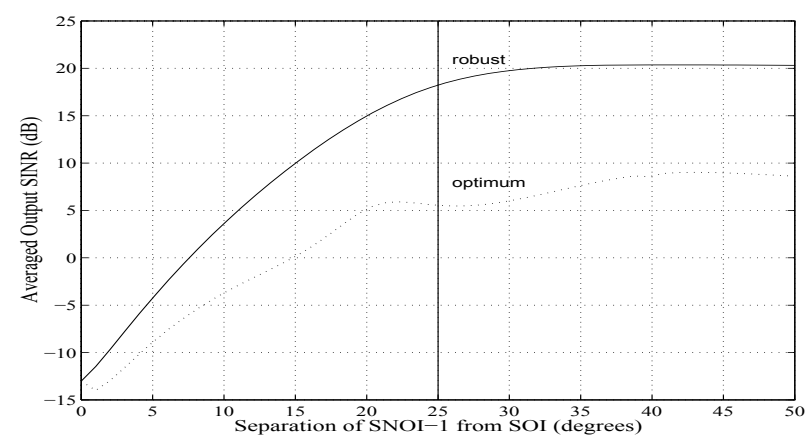

Figure 4: Output SINR vs. SOI - SNOI-1 separation. 